\title{
The prevalence and characteristics of local allergic rhinitis in Poland*
}

\author{
Andrzej Bozek', Wojciech Ścierski², Beata Ignasiak², Jerzy Jarzab', Maciej \\ Misiolek ${ }^{2}$ \\ 'Clinical Department of Internal Disease, Dermatology and Allergology in Zabrze, Medical University of Silesia, Katowice, Poland \\ ${ }^{2}$ Clinical Department of Otorhinolaryngology and Laryngological Oncology in Zabrze, Medical University of Silesia, Katowice, \\ Poland \\ ${ }^{3}$ Outpatient Clinic PMR, Swietochlowice, Poland
}

Rhinology 57: 3, $213-218,2019$

https://doi.org/10.4193/Rhin18.137

*Received for publication:

July 21, 2018

Accepted: November 20, 2018

Background: Local allergic rhinitis (LAR) is found in some patients with typical symptoms but who have negative skin prick tests and negative IgE to aeroallergens while presenting with positive nasal provocation tests for proper allergens. Little information about the clinical characteristics and prevalence of LAR has been published. The aim of this study was to determine the prevalence and characteristics of LAR in patients with symptoms of chronic rhinitis.

Methods: In total, 680 patients out of 3400 pre-screened subjects with chronic rhinitis who were at least 5 years old were included from 17 sites in Poland in the study protocol. The following medical history and diagnostic procedures were performed with aeroallergens: skin prick tests, allergen specific serum IgE and nasal provocation tests. In addition to LAR, allergic rhinitis (AR) and non-allergic (NAR) rhinitis were explored and compared.

Results: In total, 621 patients were examined. LAR was diagnosed in 109 (17.6\%) patients; AR was diagnosed in 251 (40.4\%) patients; and NAR was diagnosed in 261 (42\%) patients. In the LAR group, younger, non-smoker patients with allergies to D. pteronyssinus or grass pollen were predominant. Polysensitization was more prevalent in AR patients than in LAR patients. Bronchial asthma was at a similar level in patients diagnosed with AR (38\%) and LAR (35\%) but was significantly less prevalent in patients diagnosed with NAR (16\%). The mean age of disease onset was similar between patients with AR and LAR (17.6 $\pm 4.8 \mathrm{yrs})$, and it was significantly lower than that in patients with NAR $(24.5 \pm 6.9 \mathrm{yrs}, \mathrm{p}<0.05)$.

Conclusions: LAR is a significantly understudied problem in patients of various ages with chronic nasal symptoms. Patients with LAR and AR have similar clinical phenotypes.

Key words: local allergic rhinitis, lgE, atopy

\section{Introduction}

Chronic rhinitis continues to be a significant problem that decreases the quality of life of many people ${ }^{(1)}$. Despite typical nasal symptoms and diagnostic evidence for specific types of rhinitis, many patients have problems discerning that causes of the nasal symptoms. Some patients present with typical allergic symptoms but without allergy test confirmation. The presence of local allergic rhinitis as a new type of disease has been widely discussed in the literature for several years ${ }^{(2-4)}$. Local allergic rhinitis (LAR) is characterized by the local production of slgE during natural exposure to aeroallergens. These patients have negative skin prick tests and serum-specific lgE levels but have positive nasal provocation tests for aeroallergens ${ }^{(2,3)}$. More than $50 \%$ of patients with chronic non-allergic rhinitis may have a problem without an LAR diagnosis ${ }^{(2,5)}$. However, there is still not enough information about the prevalence of this condition.

The aim of this study was to determine the prevalence and characteristics of LAR in patients with symptoms of chronic rhinitis.

\section{Material and Methods}

Study design

A cross-sectional observational study was undertaken to determine the prevalence, clinical characteristics, severity and comorbidities of LAR in a Polish population. 


\section{Patients}

The recruitment of patients was conducted at 17 sites representative in the central, southern and northern areas of Poland. These sites consisted of 9 ENT and 8 allergy outpatient clinics. At each site, the medical base of patients was analysed according to the diagnosis or suspicion of rhinitis based on medical history and/or the use of code ICD-10 (J30-J34). With each database, patients were recruited in comparable amounts to rural and urban centres. These groups were homogeneous regarding the age and sex ratio that was consistent with the demographic structure of the Polish population in 2017. At each of the sites, approximately 200 patients were pre-screened (according to their medical history, medical data including CT scans, endoscopy results, and previous treatments), and then 50 participants were randomly selected and invited to participate in diagnostic procedures.

The number of included patients was based on a power calculation that took into account the expected effect size, the standard deviation of the outcomes and the ordinal variable for the comparative study.

The randomization procedure with random selection relied on the use of computer-generated numbers by means of a flip-coin generator (Excel, version 14.3.0, 2015, Microsoft Corporation, US).

The inclusion criteria were as follows:

- $\quad$ aged 5 years or older;

- $\quad$ provided consent to participate in the study;

- had mild, moderate or severe persistent or intermittent rhinitis according to the Allergic Rhinitis and its Impact on Asthma (ARIA) guidelines.

The exclusion criteria were as follows:

- clinical exacerbation of chronic rhinosinusitis or respiratory infections within 4 weeks prior to the study initiation;

- nasal polyposis (which is contraindicated for nasal provocation) or other serious diseases or chronic unstable disease; - nose deformity.

However, patients with chronic stable rhinosinusitis were included in the study because approximately $80 \%$ of the patients had an underlying allergy (6). The patient characteristics are presented in Table 1.

Ultimately, 680 individuals were recruited from 3400 pre-screened patients. Screening was performed by ENT doctors. During the ENT examination (see protocol), some abnormalities were observed as follows: significant septal deviation in 21 patients, turbinate hypertrophia in 38 patients, adenoids in 12 patients, nasal polyps in 24 patients, and choanal atresia in 11 patients. Some of these patients were also excluded. Finally, 621 patients were included for further study: 342 women with a mean age of $29.5 \pm 8.3$ years and 279 men with a mean age of $32.5 \pm 6.2$ years The groups were comparable in terms of numbers, gender and age (Table 1). The study was performed in 2016-2017. All the centres obtained permission to publish the data. All patients gave consent to participate in the study. The study was approved by the Bioethics Committee. After the initial recruitment, the subjects were divided into subgroups of the following age ranges: 5-18 years and older than 18 years of age.

\section{Study protocol}

Patients who met the inclusion criteria were selected to undergo further procedures as follows: medical examinations, skin prick tests with inhalant allergens (SPT), serum total IgE and specific $\lg \mathrm{E}$ antibody levels (slgE), and nasal provocation tests when the SPT and slgE were negative.

\section{Rhinitis severity}

The severity of ocular and nasal symptoms, including obstruction, rhinorrhoea (watery, mucous, and purulent), itching, and sneezing, was recorded using a visual analogue scale (VAS) of 10 $\mathrm{cm}$. Each symptom was categorized as 'mild' (VAS: 0-30 cm), 'moderate'(VAS: $>30 \mathrm{~cm}$ and $\leq 70 \mathrm{~cm})$, or 'severe' $(\text { VAS }>70 \mathrm{~cm})^{(1)}$.

\section{Medical examination}

A full rhinolaryngological examination was performed using anterior and posterior rhinoscopy and in some patients, an endoscopy and CT scan were performed.

Rhinitis was classified according to the following Allergic Rhinitis and its Impact on Asthma (ARIA) guidelines: Rhinitis is considered persistent when symptoms are present for $>4$ days/week or persist for $>4$ consecutive weeks. The rhinitis severity was based on estimations of activity impairment (sleep, daily activities, work/school performance and troublesome behaviour) and was classified as severe, moderate or mild ${ }^{(1)}$.

\section{Skin prick test (SPT)}

The SPT was performed using a panel of the following aeroallergens: D. pteronyssinus, D. farinae, Phleum pratense, Artemisia, birch, alder, hazel, Alternaria, Cladosporium, Aspergillus, cockroach and dog and cat epithelia (Allergopharma, Reinbek, Poland). A positive control (10 mg/ml histamine) and a negative control (saline) were included. An allergic reaction was defined as a positive skin test for at least one allergen, with a maximum wheal diameter of at least $3 \mathrm{~mm}$ greater than that of the negative control. Patients who did not exhibit a reaction to histamine were excluded from further analyses ${ }^{(7)}$.

\section{Serum and specific $\lg E(s \lg E)$}

Serum total and slgE antibody levels to the same aeroallergens as used in the SPT panel were determined using a fluoroenzyme immunosorbent assay (UniCAP, Uppsala, Sweden). The positive cut-off value for slgE levels was $>0.35 \mathrm{kU} / \mathrm{l}$. 
Table 1. Characteristics of all analysed patients.

\begin{tabular}{|c|c|c|c|c|c|c|}
\hline & Pre-screened group & Final study group & & dy subgro & & \\
\hline & $\mathrm{n}=\mathbf{3 4 0 0}(\%)$ & $n=621(\%)$ & $\begin{array}{c}\text { AR } \\
n=251\end{array}$ & $\begin{array}{c}\text { LAR } \\
n=109\end{array}$ & $\begin{array}{c}\text { NAR } \\
n=261\end{array}$ & p-value \\
\hline age & $28.5 \pm 6.1$ & $26.2 \pm 8.8$ & $27.1 \pm 8.3$ & $25.9 \pm 9.1$ & $38.8 \pm 7.2$ & 0.07 \\
\hline 5-18 (\%) & $1674(49.2)$ & $293(47.2)$ & $141(56.2)$ & $34(31.2)$ & $118(45.2)$ & 0.05 \\
\hline$>18(\%)$ & $1726(50.8)$ & $328(52.8)$ & $110(43.8)$ & $75(68.8)^{\wedge}$ & $143(54.8)$ & 0.04 \\
\hline women (\%) & $1789(52.6)$ & $342(55.1)$ & $129(51.4)$ & $51(46.8)$ & $162(62.1)$ & 0.07 \\
\hline urban (\%) & $2517(74)$ & $438(70.5)$ & $180(71.5)$ & $76(69.7)$ & $182(69.7)$ & 0.37 \\
\hline current or ex-smokers & $1802(53)$ & $318(51.2)$ & $102(40.6)$ & $40(36.7)$ & $176(67.4)^{*}$ & 0.06 \\
\hline atopy in family & $1730(50.8)$ & $300(48.3)$ & $131(52.9)$ & $55(50.5)$ & $114(43.7)$ & 0.29 \\
\hline
\end{tabular}

AR - allergic rhinitis, LAR - local allergic rhinitis, NAR - non-allergic rhinitis; There were no significant differences in any of the parameters between the pre-screened and final study groups. ${ }^{*}$; -value for the comparison of the three sub-groups; $\wedge$ - significant differences between LAR and AR and between LAR and NAR $(p<0.05)$.

\section{Nasal provocation test (NPT)}

Nasal provocation tests were performed using acoustic rhinometry with an SRE 2000 rhinometer (Rhinometrics, Lynge, Denmark). These tests were performed according to the guidelines of the Standardization Committee on Acoustic Rhinometry and the EAACl position paper ${ }^{(8,9)}$. The nasal provocation tests were performed when the concentrations of the examined allergens were lower in Poland. First, using a metered pump spray, the patients were intranasally challenged with saline to exclude nasal hyper-reactivity. If the nasal provocation test was negative, it was performed again one week later with another provocation with saline as the negative control and then with the NPT with extracts: D. pteronyssinus, Phleum pratense, Artemisia, birch and cat (the concentration of each allergen was $5000 \mathrm{SBE} / \mathrm{mL}$ (Allergopharma, Reinbek, Germany). A total of $100 \mathrm{~mL}$ of the solution of allergen was applied to each nostril. Two additional NPTs were performed with one of the other extracts at one-week intervals. The order of allergen provocation was as stated for the abovementioned aeroallergens. The total volume of both nasal cavities was determined to be $2-6 \mathrm{~cm}$ using acoustic rhinometry, and the results were compared with the baseline test. The immediate reaction was analysed at $15 \mathrm{~min}, 1$ hour and 6 hours according to the protocol based on the EAACl position paper ${ }^{(9)}$.

\section{NPT monitoring}

Nasal symptoms as a total score (without analysing individual symptoms) were monitored using the $100-\mathrm{mm}$ horizontal visual analogue scale (VAS). Symptom severity was classified as severe (71-100), moderate (31-70) or mild (0-30). The responses were monitored at baseline, $15 \mathrm{~min}$ after saline application, and 15 $\min , 1$ hour and 6 hours after allergen application. The response was determined using a VAS, and the total normal volume of the nasal cavity was $2-6 \mathrm{~cm}$. An increase $\geq 30 \%$ in the total VAS score and/or a decrease $\geq 30 \%$ in the total VAS score bilaterally in the nasal volume (normal, 2-6 cm) were considered positive responses (in any of the analysed times after the challenge). Patients were classified into three groups according to the clinical test results:

- allergic rhinitis: positive SPT and/or slgE

- local allergic rhinitis: negative SPT and slgE and positive NPT - non-allergic rhinitis: negative SPT, slgE and NPT.

\section{Lung function test}

Spirometry was performed using a Lungtest 1000 spirometer (MES, Cracow, Poland). A positive reversibility test was defined as an increase in FEV1.0 $\geq 12 \%$ and $\geq 200 \mathrm{ml}$ in relation to baseline after inhalation of $400 \mathrm{mcg}$ of salbutamol.

\section{Statistical analysis}

Statistica programme, version 8.0 (StatSoft, Poland) was used for all statistical analyses. A p-value $<0.05$ was considered to be statistically significant. Descriptive analyses were performed and expressed as the means and standard deviation. Non-parametric statistical analyses were performed using the chi-square test and multivariate ANOVA.

\section{Results}

\section{Patients characteristics}

In total, 621 patients underwent the protocol and were used in the final analysis (59 patients were excluded because of a deviation from the protocol or an incomplete protocol). Patient characteristics are presented in Table 1. LAR was confirmed in 109 (17.6\%) patients; AR was confirmed in 251 (40.4\%) patients; and NAR was confirmed in 261 (42\%) patients. Based on all 3,400 pre- screened group, LAR was observed in $3.3 \%$ patients with a suspicion of rhinitis or diagnosis of rhinitis. 
Table 2. Distribution of different features of rhinitis in the study group.

\begin{tabular}{|c|c|c|c|c|}
\hline rhinitis & AR & LAR & NAR & p-value \\
\hline \multicolumn{5}{|c|}{$\begin{array}{l}\text { Mean nasal } \\
\text { symptoms score } \\
\text { using VAS } \pm \text { SD } \\
\text { baseline: }\end{array}$} \\
\hline & $20.4 \pm 10.6$ & $22.9 \pm 10.3$ & $29.7 \pm 20.2$ & 0.19 \\
\hline congestion & $33.1 \pm 17.6$ & $29.1 \pm 32.1$ & $57.4 \pm 32.7$ & 0.04 \\
\hline rhinorrhoea & $25.4 \pm 9.1$ & $28.4 \pm 11.1$ & $21.5 \pm 9.9$ & 0.15 \\
\hline sneezing & $18.4 \pm 6.1$ & $15.5 \pm 8.4$ & $7.8 \pm 4.3$ & 0.05 \\
\hline mild \% & 18 & 22 & 16 & 0.08 \\
\hline moderate $\%$ & 52 & 48 & 51 & 0.23 \\
\hline severe $\%$ & 30 & 30 & 33 & 0.31 \\
\hline intermittent \% & 31 & 29 & 23 & 0.20 \\
\hline persistent $\%$ & 69 & 71 & 77 & 0.42 \\
\hline seasonal \% & $42^{\wedge}$ & 24 & 28 & 0.08 \\
\hline perennial \% & $43 \wedge$ & 74 & 72 & 0.05 \\
\hline
\end{tabular}

AR - allergic rhinitis, LAR - local allergic rhinitis, NAR - non allergic rhinitis; p-value for the comparison of the three groups.

The LAR group predominantly consisted of non-smokers and younger patients compared to the NAR group.

No correlation was observed among the regions of Poland and the prevalence of AR, LAR and NAR.

Rhinitis symptoms at baseline and concomitant allergy

The profile of clinical symptoms (according to the VAS) in different types of rhinitis in the study group is shown in Table 2 . The severity of rhinitis was similar in all groups. However, intermittent rhinitis was rarely noticed in the AR group in comparison to the other groups (Table 2). The mean duration of the analysed forms of rhinitis was as follows: $8.6 \pm 4.6 \mathrm{yrs}$ for AR vs. $9.3 \pm 5.9 \mathrm{yrs}$ for LAR vs. $9.8 \pm 6.3$ yrs for NAR. No significant differences were observed. The mean age of disease onset was similar between patients with AR and LAR (17.6 $\pm 4.8 \mathrm{yrs})$; it was significantly higher in patients diagnosed with NAR than in the other groups (24.5 \pm 6.9 yrs, $\mathrm{p}<0.05)$.

Bronchial asthma was present at similar levels in AR patients (38\%) and LAR patients (35\%) but was significantly less prevalent in NAR patients (16\%). The most common allergens that provoked a positive reaction included D. pteronyssinus (52\%) and Phleum pratense (47\%) in AR patients for the SPT and D. pteronyssinus (65\%) and grass (41\%) in the NPT in LAR patients. Polysensitisation was more prevalent in AR patients than in LAR patients. The results are presented in Table 3.

\section{Nasal provocation tests}

The results of the NPT are shown in Table 4. There were no positive results of the NPT in patients with NAR; however, a positive reaction to saline was observed in 5 patients.

The profile and number of positive results of the NPT were
Table 3. Results of allergy diagnostics.

\begin{tabular}{|c|c|c|c|c|}
\hline & $\begin{array}{c}\text { AR } \\
n=251\end{array}$ & $\begin{array}{c}\text { LAR } \\
n=109\end{array}$ & $\begin{array}{c}\text { NAR } \\
n=261\end{array}$ & p-value \\
\hline asthma (\%) & $95(38)$ & $38(35)$ & $42(16)$ & 0.11 \\
\hline atopic dermatitis & $23(9.2)$ & $9(8.2)$ & - & 0.45 \\
\hline oral syndrome & 89 (35.6) & $7(6.4)^{*}$ & - & 0.02 \\
\hline chronic urticaria & $18(7.2)$ & $8(9.2)$ & $21(8)$ & 0.38 \\
\hline conjunctivitis & $154(61)$ & $51(46.8)$ & $45(17.2)$ & 0.03 \\
\hline $\begin{array}{l}\text { NSAID hypersen- } \\
\text { sitivity }\end{array}$ & 34 (13.5) & $12(11.1)$ & 39 (14.9) & 0.26 \\
\hline $\begin{array}{l}\text { Total serum lgE } \\
(\mathrm{kU} / \mathrm{L})\end{array}$ & $178.8 \pm 96.4$ & $63.9 \pm 11.9$ & $48.8 \pm 33.8$ & 0.05 \\
\hline \multicolumn{5}{|l|}{ in SPT } \\
\hline $\begin{array}{l}\text { D. pteronyssinus } \\
(\%)\end{array}$ & $130(52)$ & 0 & 0 & - \\
\hline D. farinae (\%) & $119(47.4)$ & 0 & 0 & - \\
\hline $\begin{array}{l}\text { Phleum pratense } \\
\text { (\%) }\end{array}$ & $118(47)$ & 0 & 0 & - \\
\hline Artemisia (\%) & $35(4)$ & 0 & 1 & - \\
\hline Alternaria (\%) & $27(10.8)$ & 0 & 0 & - \\
\hline birch (\%) & $89(35.5)$ & 0 & 0 & - \\
\hline alder (\%) & $34(13.5)$ & 0 & 0 & - \\
\hline hazel (\%) & $46(18.3)$ & 0 & 0 & - \\
\hline cat (\%) & $11(4.4)$ & 0 & 0 & - \\
\hline $\begin{array}{l}\text { polysensitisation } \\
\text { (\%) }\end{array}$ & $87(34.7)$ & 0 & 0 & - \\
\hline
\end{tabular}

AR - allergic rhinitis, LAR - local allergic rhinitis, NAR - non allergic rhinitis, SPT: skin prick test, NPT - nasal provocation test; $\mathrm{p}$-value for the comparison of the three groups; * - significant differences between AR and. LAR $(p<0.05)$

similar between the AR and LAR groups except for Artemisia and birch allergens.

\section{Discussion}

This study shows that the prevalence of LAR is a significant problem in Polish populations. The strengths of this paper include the fact that this population was one of the largest populations that has been tested in this way. The prevalence of LAR determined in this study falls within the results of previous studies: $7.5 \%$ in a Chinese population and $25.7 \%$ in a Spanish population $(4,10)$. Additionally, there were many analyses on a small group of patients from one site ${ }^{(11-15)}$.

It seems that LAR is still an unappreciated type of rhinitis. Most of the analysed patients had an incorrect initial diagnosis prior to this study. In addition, the time between the first symptoms and the diagnosis was long in duration, i.e., $>2-3$ years (these data were not complete and therefore were not presented in the results). It is important to show that LAR has many similarities 
Table 4. Results of the NPT in the study group.

\begin{tabular}{|c|c|c|c|c|c|c|}
\hline \multirow[t]{2}{*}{ allergen } & \multicolumn{2}{|c|}{ number of positive results $\wedge$} & \multicolumn{2}{|c|}{$\begin{array}{c}\text { mean percentage decrease of nasal } \\
\text { volume after NPT }\end{array}$} & \multicolumn{2}{|c|}{$\begin{array}{c}\text { mean total VAS score }(95 \% \mathrm{Cl}) \text { after } \\
\text { NPT }\end{array}$} \\
\hline & $A R n=251(\%)$ & LAR $n=109(\%)$ & $A R n=251(\%)$ & LAR $n=109(\%)$ & $A R n=251(\%)$ & LAR $n=109(\%)$ \\
\hline D. pteronyssinus & $157(62.5)$ & $71(65)$ & 47.9 & 53.9 & $70(58 ; 72)$ & $65(57 ; 74)$ \\
\hline Phleum pratense & $93(37.1)$ & $45(41)$ & 57.3 & 52.1 & $74(54 ; 92)$ & $69(52 ; 84)$ \\
\hline Artemisia & $43(17)^{*}$ & $5(4.6)$ & $43.2^{*}$ & 66.5 & $71(56 ; 85)$ & $77(55 ; 92)$ \\
\hline birch & $25(9.9)^{*}$ & $5(4.6)$ & 49.3 & 40.3 & $65(39 ; 82)$ & $55(38 ; 71)$ \\
\hline cat & $15(6)$ & $6(5.5)$ & 40.1 & 44.8 & $74(67 ; 91)$ & $78(55 ; 94)$ \\
\hline
\end{tabular}

AR - allergic rhinitis, LAR - local allergic rhinitis; ^ positive results based on criteria of a positive NPT: increase $\geq 30 \%$ in the total VAS score and/or a decrease in the total VAS $\geq 30 \%$ bilaterally in the nasal volume (normal nasal volume $2-6 \mathrm{~cm}$ ); * significant differences between the AR and LAR groups.

with other types of rhinitis including the following: severity, mean age of appearance, presence of atopy in the family and percentage of hypersensitivity to NSAIDs. These similarities are consistent with that described by other authors ${ }^{(4)}$.

Additionally, there are many differences. A typical patient with LAR is an older man with more perennial types of symptoms who is monosensitized and more hypersensitive to $D$. pteronyssinus. This finding is in contrast with that described by Rondon et al., who reported that LAR was more predominant in women (4). However, their observations that LAR and AR had a similar profile were also observed in the present study.

It is very important to emphasize that LAR can be present in children older than 5 years of age, which has been confirmed by other authors ${ }^{(4,16,17)}$. Thus, consideration should be given to diagnoses in young patients.

Patients with LAR have the same profile of comorbidities as patients with AR, as confirmed by other authors ${ }^{(4)}$.

The common problem with distinguishing patients with LAR from those with NAR is related to the fact that the severity of rhinitis, the presence of atopy in family members, a low level of total serum IgE, negative skin prick tests and urban living were similar.

The clinical profile of NAR patients was similar to other observations ${ }^{(4,5,18,19)}$. However, it is interesting to note that the NAR patients had a lower risk of asthma in comparison to other groups. The weakness of this paper is its lack of patients who could have had LAR during screening but were excluded based on the exclusion criteria. The limitation of the study was that the group of LAR patients was small in size, which prevented us from obtaining a more comprehensive clinical picture. Additionally, the distinction of patients who use the NPT could be limited based on the sensitivity and specificity of this tool. The use of the nasal fraction for lgE monitoring after provocation is recommended; however, this approach is too time consuming for cohort studies.

\section{Conclusions}

LAR is a frequent problem in patients of various ages with chronic nasal symptoms. Patients with LAR and AR have similar clinical phenotypes. D. pteronyssinus was observed to be the most sensitising allergen in patients with LAR.

\section{Acknowledgements}

None.

\section{Authorship contribution}

$A B, W S, B I$ and JJ recruited patients and obtained the data. $A B$, WS, and MM managed the clinical trial, and BK reviewed the literature and drafted the manuscript. All the authors critically reviewed the manuscript, approved and accepted the final version for submission.

\section{Conflict of interest}

No conflict of interest.

\section{References}

1. Bousquet J, Khaltaev N, Cruz AA, Denburg J, Fokkens WJ, Togias A et al.World Health Organization.; GA(2)LEN: Allergic rhinitis and its impact on asthma (ARIA 2008). Allergy. 2008; 63 (supp86): 8-160.

2. Rondon C, Romero JJ, Lopez A, Antúnez C, Martín-Casañez E, Torres MJ et al. Local lgE production and positive nasal provocation test in patients with persistent nonallergic rhinitis. J Allergy Clin Immuno.I 2007; 119: 899-905.

3. Carney AS, Powe DG, Huskisson RS, Jones NS. Atypical nasal challenges in patients with idiopathic rhinitis: more evidence for the existence of allergy in the absence of atopy? Clin Exp Allergy. 2002; 32: 1436-4140. 4. Rondon C, Campo P. Eguilaz-Gracia I, Plaza C, Bogad D, et al. Local allergic rhinitis is an independent rhinitis phenotype: The results of a 10-year follow-up study. Allergy. 2018; 73(2):470-478

5. Rondon C, Campo P, Galindo L, BlancaLópez N, Cassinello MS, Rodriguez-Bada 
$J$ et al. Prevalence and clinical relevance of local allergic rhinitis. Allergy. 2012; 67: 1282-1288

6. Mosges R, Stierna P. Why do we treat allergies antibiotics? Curr Opin Allergy Clin Immunol. 2006; 6(3): 144-145.

7. Bousquet J, Heinzerling L, Bachert C, Papadopoulos NG, Bousquet PJ, Burney PG et al. ; Global Allergy and Asthma European Network: Practical guide to skin prick tests in allergy to aeroallergens. Allergy. 2013 67: 18-24

8. Hilberg $\mathrm{O}$, Pedersen OF. Acustic rhinometry: recommendations for technical specifications and standard operating procedures Rhinology. 2000; 16: 3-17.

9. Auge J, Vent J, Agache I, Airaksinen L, Campo Mozo P, Chaker A, et al. EAAC Position Paper on the standardization of nasal allergen challenges. Allergy. 2018; 8: 1597-1608.

10. Cheng KJ, Xu YY, Liu HY, Wang SQ. Serum eosinophil cationic protein level in Chinese subjects with nonallergic and local allergic rhinitis and its relation to the severity of disease. Am J Rhinol Allergy. 2013; 27:8-12.

11. Arasi S, Pajno GB, Lau S, Matricardi PM. Loca allergic rhinitis - a critical reappraisal from a paediatric perspective. Pediatr Allergy Immunol. 2016; 27:569-573.

12. Campo P, Salas M, Blanca-López N, Rondón C. Local allergic rhinitis. Immunol Allergy Clin North Am. 2016;36:321-332

13. Powe DG, Huskisson RS, Carney AS, et al. Evidence for an inflammatory pathophysiology in idiopathic rhinitis. Clin Exp Allergy. 2001; 31:864-872.

14. Roca F, De la, Blanca-Lopez N, Rondon C, et al. Seasonal local allergic rhinitis in areas with high exposure to grass pollen. J Allergy Clin Immunol. 2012; 129:AB111.

15. Cruz Niesvaara D, Rondon C, Almeida Quintana L, et al. Evidence of local allergic rhinitis in areas with high and permanent aeroallergens exposure. J Allergy Clin Immunology. 2012; 129:AB111.

16. Jung CG, Lee JH, Ban GY, Park HS, Shin YS Prevalence and Clinical Characteristics of Local Allergic Rhinitis to House Dust Mites. Yonsei Med J. 2017;58(5):1047-1050. doi: 10.3349/ymj.2017.58.5.1047.

17. Ha EK, Na MS, Lee S, Baek H, Lee SJ Sheen $\mathrm{YH}$ et al. Prevalence and Clinical Characteristics of Local Allergic Rhinitis in Children Sensitized to House Dust Mites. Int Arch Allergy Immunol. 2017;174(3-4):183-
189

18. Hellings PW, Klimek L, Cingi C, Agache Akdis C, Bachert C, et al. Non-allergic rhinitis: Position paper of the European Academy of Allergy and Clinica Immunology. Allergy. 2017;72(11):1657-65.

19. Rondon C, Bogas G, Barrionuevo E, Blanca M, Torres MJ, Campo P. Nonallergic rhinitis and lower airway disease. Allergy. 2017;72(1):24-34.

Andrzej Bozek

Clinical Department of Internal

Disease

Dermatology and Allergology Medi-

cal University of Silesia

41-800 Zabrze

MC Sklodowskiej 10

Poland

Tel/Fax: +48 322713165

E-mail: andrzejbozek@o2.pl 